\title{
Un caso de neumomediastino espontáneo que simula un síndrome de Boerhaave*
}

\author{
Drs. ANA BELÉN ALÁEZ CH. ${ }^{1}$, JULIO GALINDO A. ${ }^{1}$, JAVIER PÁRAMO Z. ${ }^{1}$, \\ PEDRO CARDA A. ${ }^{1}$, CARMEN RAMIRO P. ${ }^{1}$, NURIA LOSA B. ${ }^{1}$, JOSÉ NISTAL P. ${ }^{1}$
}

1 Servicio de Cirugía General y del Aparato Digestivo del Hospital Universitario Ramón y Cajal, Madrid, España.

\section{Abstract}

\section{A case of spontaneous pneumomediastinum that simulates a Boerhaave's syndrome}

The spontaneous pneumomediastinum is the presence of air inside the mediastinum without a traumatic or iatrogenic reason associated. It is a slightly frequent entity and as the spontaneous pneumotorax it is given especially in young males. In rare occasions it associates to an episode of vomits and in this case it is necessary to make a differential diagnosis with Boerhaave's syndrome because at the first moment the clinic can be similar in both cases. Nevertheless, while the spontaneous pneumomediastinum is quite often to be a benign entity with good prediction, Boerhaave's syndrome has a mortality of $80 \%$ $90 \%$ if the diagnosis is produced after $24-48$ hours.

Key words: Spontaneous pneumomediastinum, subcutaneous emphysema.

\section{Resumen}

El neumomediastino espontáneo es la presencia de aire dentro del mediastino sin que exista una causa traumática o iatrogénica asociada. Es una entidad poco frecuente que, al igual que el neumotórax espontáneo, se da sobre todo en varones jóvenes. En raras ocasiones se asocia a un episodio de vómitos $\mathrm{y}$, en este caso, es necesario hacer un diagnóstico diferencial con el síndrome de Boerhaave ya que en un primer momento la clínica puede ser similar en ambos cuadros. Sin embargo, el neumomediastino espontáneo suele ser una entidad benigna con buen pronóstico y el síndrome de Boerhaave es un cuadro de mal pronóstico y que, de diagnosticarse después de 24-48 horas, puede tener hasta un 80\%-90\% de mortalidad.

Palabras clave: Neumomediastino espontáneo, enfisema subcutáneo.

\footnotetext{
*Recibido el 30 de Mayo de 2008 y aceptado para publicación el 28 de Julio de 2008.

Correspondencia: Dra. Ana Belén Aláez C.

Calle Almansa, $58,2^{\circ} \mathrm{F}$.

28039, Madrid, España

E-mail: anabelenalaez@hotmail.com
} 


\section{Introducción}

El neumomediastino espontáneo es la existencia de aire en el mediastino que no está asociada a causa traumática ni iatrogénica. Se suele dar en varones jóvenes con antecedentes de asma. Ocurre por un aumento de la presión intraalveolar que provoca la rotura de los mismos en su base y causa una salida del aire al espacio intersticial hacia el hilio, desarrollando así el neumomediastino o el neumotórax. Rara vez el neumomediastino espontáneo se produce por un episodio de vómitos. En el caso en el que se asocie a vómitos se debe hacer un diagnóstico diferencial con el síndrome de Boerhaave, caracterizado por la rotura espontánea del esófago asociada a aumentos bruscos de la presión intraabdominal ${ }^{1}$.

\section{Caso clínico}

Presentamos un caso de neumomediastino espontáneo que asemejaba a un síndrome de Boerhaave. Se trata de una mujer de 29 años de edad, alérgica a penicilinas y sin otros antecedentes de interés, que acude al servicio de urgencias porque refiere crepitación en tórax y en cuello tras

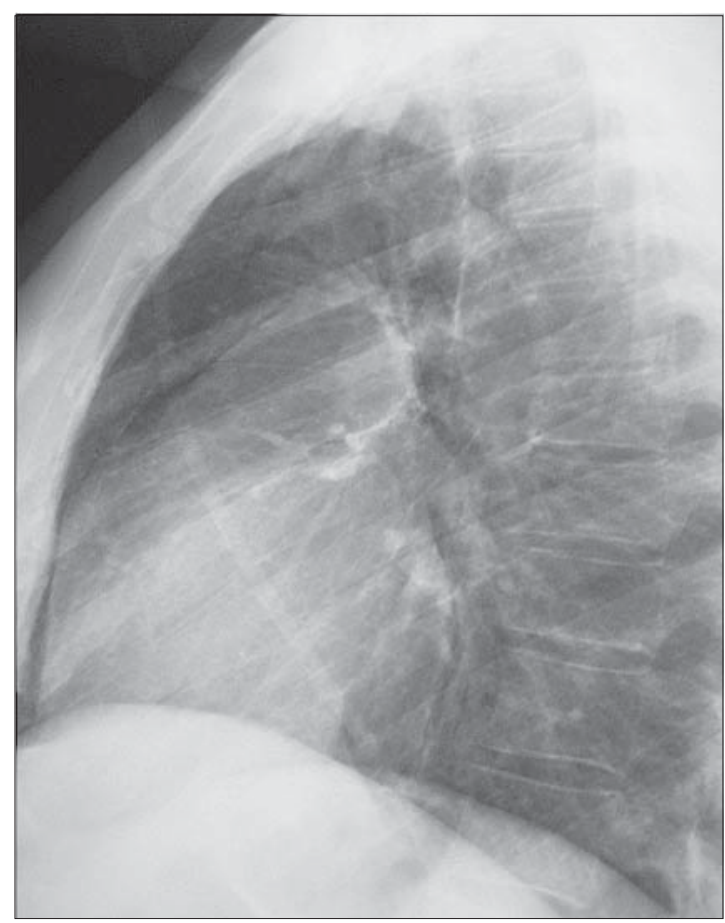

Figura 1. episodio de vómitos de repetición. También cuenta dolor torácico con la respiración profunda y con el movimiento. A la exploración física se encuentra estable hemodinámicamente (con TA: 100/75 y FC: $70 \mathrm{lpm}$ ), no sudorosa ni taquipneica. No presenta fiebre. Se palpa crepitación en cuello, mandíbula y cara anterior de tórax y axila derecha; no refiere dolor abdominal espontáneo ni a la palpación.

Se realiza analítica en la que destacan 11.600 leucocitos con neutrofilia (89\%) y resto de parámetros analíticos normales. En la radiografía de tórax se observa un neumomediastino que es más marcado en la radiografía lateral (Figura 1) que en la posteroanterior y enfisema subcutáneo.

Se sospecha que se trate de un neumomediastino espontáneo, pero debido al antecedente de vómitos de repetición se decide realizar una TAC de tórax y un esofagograma con contraste hidrosoluble para descartar un síndrome de Boerhaave. En la TAC de tórax (Figura 2) se observa neumomediastino y una pequeña cantidad de gas retrocrural con enfisema en axila derecha y cervical. No se observa fuga de contraste, derrame pleural ni otras alteraciones parenquimatosas.

El esofagograma es informado como normal y no se observa fuga de contraste.

Debido a que la paciente se encuentra con buen estado general, estable hemodinámicamente y se ha descartado un síndrome de Boerhaave, se decide ingreso en planta con antibioterapia de amplio espectro (levofloxacino y metronidazol por ser la paciente alérgica a penicilinas) y oxigenoterapia. $\mathrm{La}$ paciente presenta buena evolución clínica y radiológica en la planta; el enfisema subcutáneo va disminuyendo hasta que a los 5 días ha desaparecido por completo. Se realiza una radiografía de control

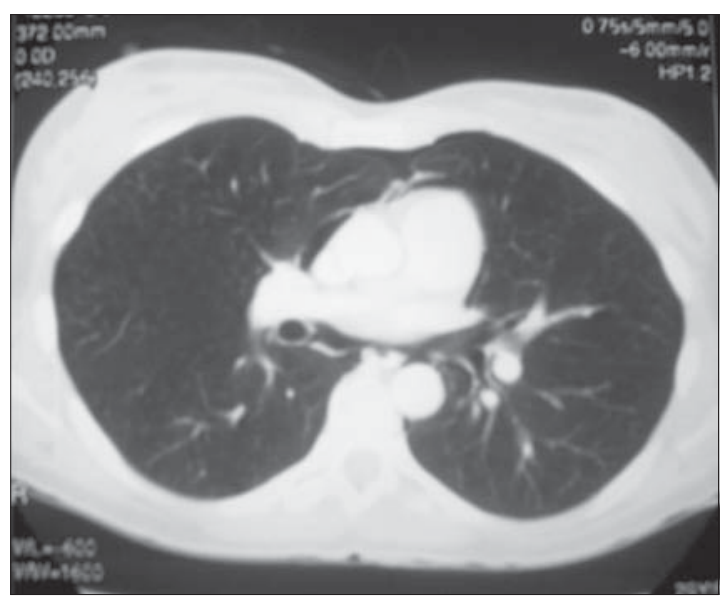

Figura 2. 
a las 48 horas en la que se sigue observando el neumomediastino, aunque no presenta derrame pleural ni ningún otro hallazgo patológico. La paciente es dada de alta al sexto día de ingreso completamente asintomática y con control radiográfico normal.

\section{Discusión}

El neumomediastino espontáneo es una entidad que se da sobre todo en varones jóvenes y constituye el $1 \%$ de los casos de neumomediastino. Son factores predisponentes el asma, las drogas inhaladas, el submarinismo y, en general, cualquier maniobra que produzca un aumento de la presión intraalveolar (vómitos, tos intensa...) $)^{2}$. En la clínica es característico el dolor torácico, la disnea y, en ocasiones, la disfagia. El enfisema subcutáneo puede aparecer hasta en el $80 \%$ de los casos ${ }^{3}$. El diagnóstico se realiza mediante la clínica y la radiografía de tórax y, en los casos en los que se sospeche un síndrome de Boerhaave, se debe realizar un esofagograma con contraste hidrosoluble que mostrará la salida del mismo desde el esófago o una TAC de tórax que, además de mostrar el neumomediastino, también evidencia la fuga del contraste. El pronóstico del neumomediastino es- pontáneo suele ser bueno y el paciente se suele recuperar en 72-96 horas con tratamiento conservador (oxigenoterapia, reposo relativo y antibioterapia profiláctica $)^{4}$.

Como conclusión podríamos decir que el neumomediastino espontáneo debido a vómitos de repetición es una causa de neumomediastino poco frecuente y que en este caso conviene realizar el diagnóstico diferencial con el síndrome de Boerhaave lo más precozmente posible, ya que el pronóstico de esta última entidad varía enormemente según el momento del diagnóstico.

\section{Referencias}

1. Calvin SH, Wilfred LM, Anthony PC, Yim MD. Barogenic esophageal rupture: Boerhaave syndrome. J Can Chir 2006; 49: 438-439.

2. Newcomb A, Clarke P. Spontaneous Pneumomediastinum. A benign curiosity or a significant problem? Chest 2005; 128: 3298-3302.

3. Miura H, Taira O, Hiraguri S. Clinical features of medical pneumomediastinum. Ann Thorac Cardiovasc Surg 2003; 9: 188-191.

4. Fibla JJ, Farina C, Gómez J. Neumomediastino espontáneo: dificultades en el diagnóstico. Arch Bronconeumol 2002; 38: 499-504. 